Supporting Information

\title{
Large-Scale Metabolite Analysis by Laser Desorption Ionization Mass Spectrometry from Silicon Nanopost Arrays
}

\author{
Andrew R. Korte ${ }^{\dagger}$, Sylwia A. Stopka ${ }^{\dagger}$, Nicholas Morris ${ }^{\ddagger}$, Trust Razunguzwa ${ }^{\ddagger}$, and Akos Vertes ${ }^{\dagger}{ }^{*}$ \\ ${ }^{\dagger}$ Department of Chemistry, George Washington University, Washington, DC 20052, USA \\ ${ }^{\ddagger}$ Protea Biosciences, Inc., Morgantown, WV 26505, USA
}

\section{*Corresponding Author}

Email: vertes@gwu.edu (A. Vertes). Phone: +1 202-994-2717. Fax: +1 202-994-5873. Address: Department of Chemistry, The George Washington University, 800 22-nd Street, N.W., Washington, DC 20052, USA. 


\section{Table of Contents}

Supplement for EXPERIMENTAL SECTION .................................................................... S-3

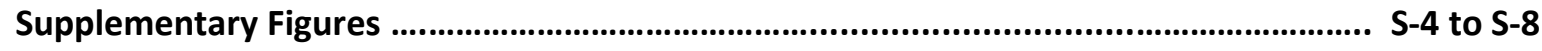

Supplementary Tables ......................................................................................................... S-9 to S-18

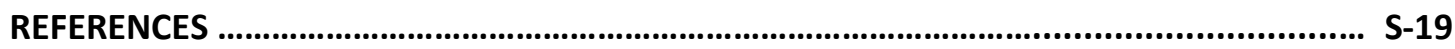

\section{IN SEPARATE FILES:}

Spreadsheet S1. All metabolite ions detected in NAPA-LDI-MS analysis of standard mixtures.

Spreadsheet S2. Detected metabolites within the metabolic pathways presented in Figure 3. 


\section{Supplement for EXPERIMENTAL SECTION}

Serum extraction and analysis. Human Serum Type AB, Male (Cat. No. H4522 and Lot No. SLBN9196V) was purchased from Sigma-Aldrich (St. Louis, MO). Proteins were precipitated from $100 \mu \mathrm{L}$ aliquots of the serum by the addition of $400 \mu \mathrm{L}$ of $-20^{\circ} \mathrm{C}$ methanol. After methanol addition, the samples were vortexed briefly to mix, sonicated for $5 \mathrm{~min}$, and incubated at $-20^{\circ} \mathrm{C}$ for $1 \mathrm{~h}$. They were then centrifuged for $10 \mathrm{~min}$ at $14,000 \times \mathrm{g}$ and $4{ }^{\circ} \mathrm{C}$.

The supernatant was transferred to a fresh sample tube, and $400 \mu \mathrm{L}$ of $-20{ }^{\circ} \mathrm{C}$ chloroform and $100 \mu \mathrm{L}$ of $4{ }^{\circ} \mathrm{C}$ water were added to each sample, inducing separation into organic and aqueous phases. The samples were then briefly vortexed and centrifuged for $10 \mathrm{~min}$ at $14,000 \times \mathrm{g}$ and $4{ }^{\circ} \mathrm{C}$. The organic and aqueous phases from each sample were removed to separate sample tubes and dried in a vacuum centrifuge at $4{ }^{\circ} \mathrm{C}$, then stored at $-80^{\circ} \mathrm{C}$ until use.

Aqueous extracts were reconstituted in $100 \mu \mathrm{L}$ of water and $0.5 \mu \mathrm{L}$ was spotted onto each nanopost array. Organic extracts were reconstituted in $10 \mu \mathrm{L}$ of 1:1 acetone:water and $0.2 \mu \mathrm{L}$ was spotted onto each nanopost array. All extract samples were analyzed using a fluence of $100 \mathrm{~mJ} / \mathrm{cm}^{2}$, three laser shots per scan, and one scan per raster point. The full area of each array was sampled at a raster pitch of $100 \mu \mathrm{m}$. Spectra were acquired in the orbitrap analyzer using a resolving power setting of 30,000, and $\mathrm{m} / \mathrm{z}$ ranges of $100-600$ for aqueous extracts and 200-1000 for organic extracts.

For standard addition experiments in which serum samples were spiked with known concentrations of metabolites, standards of glucose or an amino acid mixture (arginine, phenylalanine, and proline) were prepared in water and diluted to a range of concentrations. Concentrations of the spike solutions were selected such that final serum concentrations of the metabolites were $\sim 1 \times$ (unspiked), $\sim 1.5 \times, \sim 2 \times, \sim 3 \times, \sim 5 \times$, and $\sim 10 \times$ the reported natural concentrations ${ }^{1}$. Prior to protein precipitation, $10 \mu \mathrm{L}$ of the standard solution was added to the raw serum and samples were processed as above. 


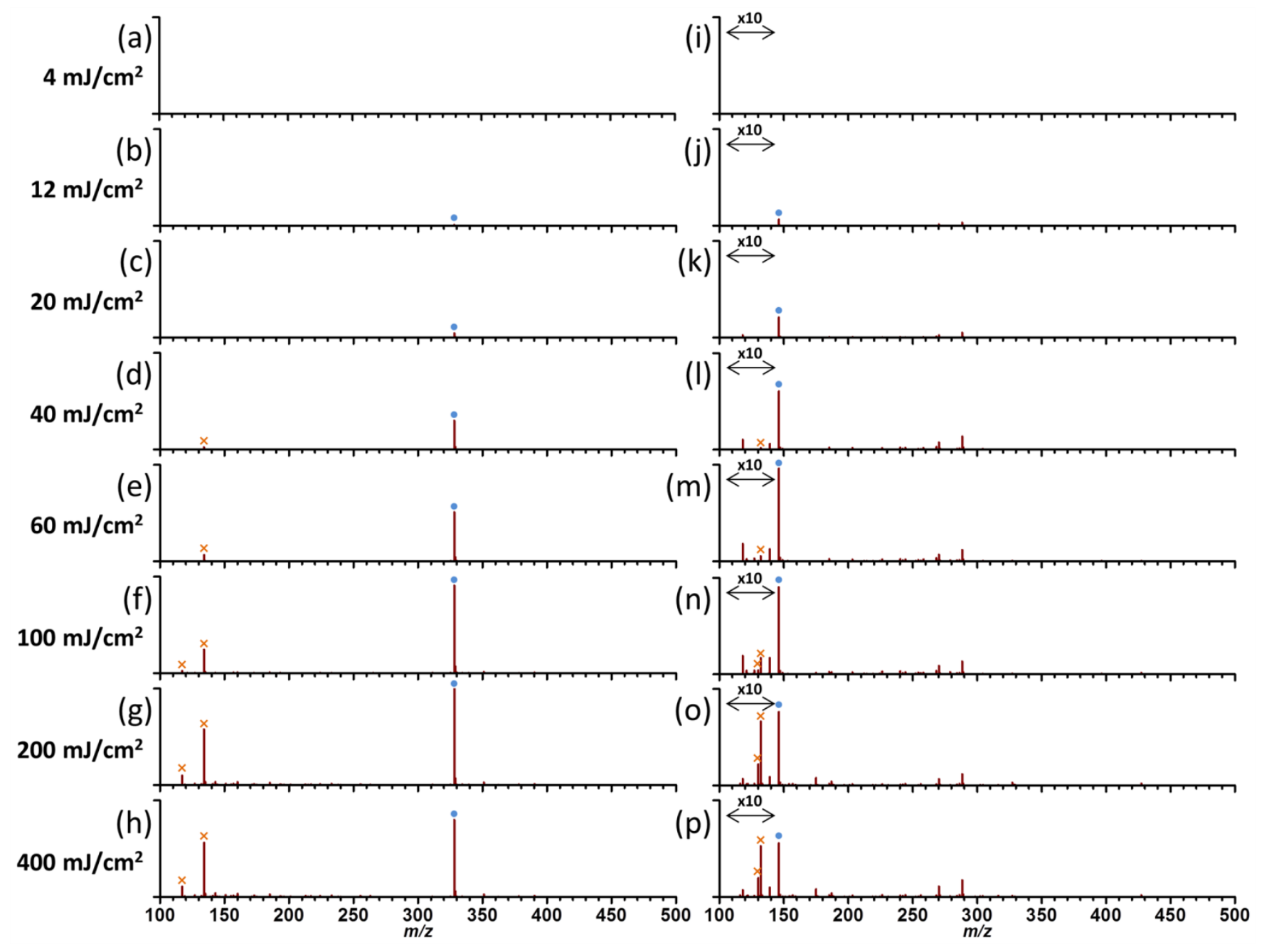

Figure S1. Mass spectra obtained from NAPA-LDI-MS of (a-h) 3',5'-cyclic adenosine monophosphate (cAMP) and (i-p) acetylcholine at a range of laser fluences. Intact metabolite ions $\left([\mathrm{M}]^{+}\right.$for acetylcholine and $[\mathrm{M}-\mathrm{H}]^{-}$for cAMP) are denoted by $(\bullet)$, whereas $(\times)$ denotes fragment ions. cAMP and acetylcholine spectra are normalized to absolute intensities of $1.2 \times 10^{6}$ au and $2.0 \times 10^{6}$ au, respectively. 


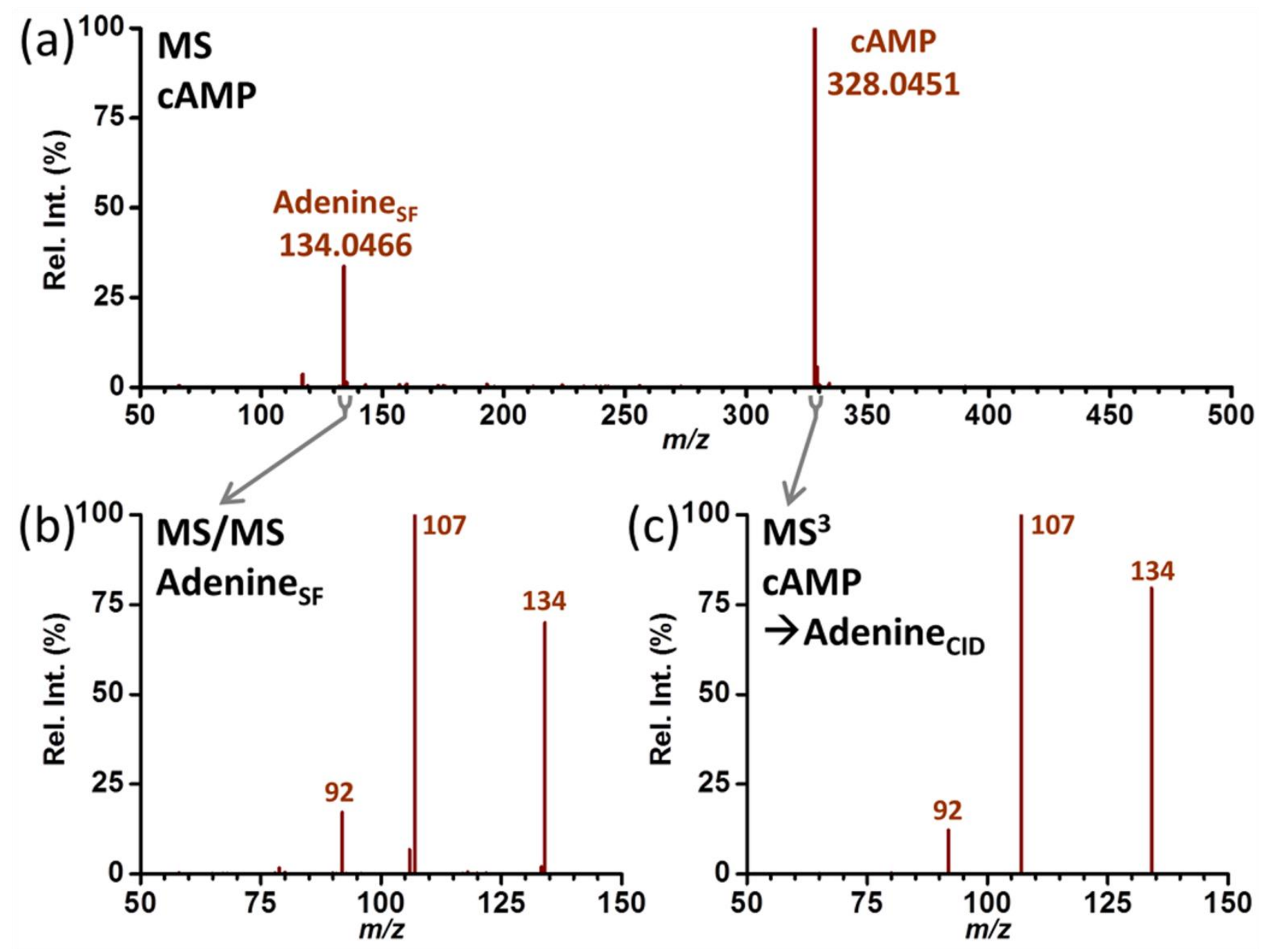

Figure S2. (a) MS spectrum of cAMP standard at $100 \mathrm{~mJ} / \mathrm{cm}^{2}$ fluence showing structure specific fragmentation (SF) to adenine ion. (b) MS/MS spectrum of adenine generated by structure specific fragmentation of cAMP. (c) $\mathrm{MS}^{3}$ spectrum of adenine generated by CID fragmentation of cAMP. 

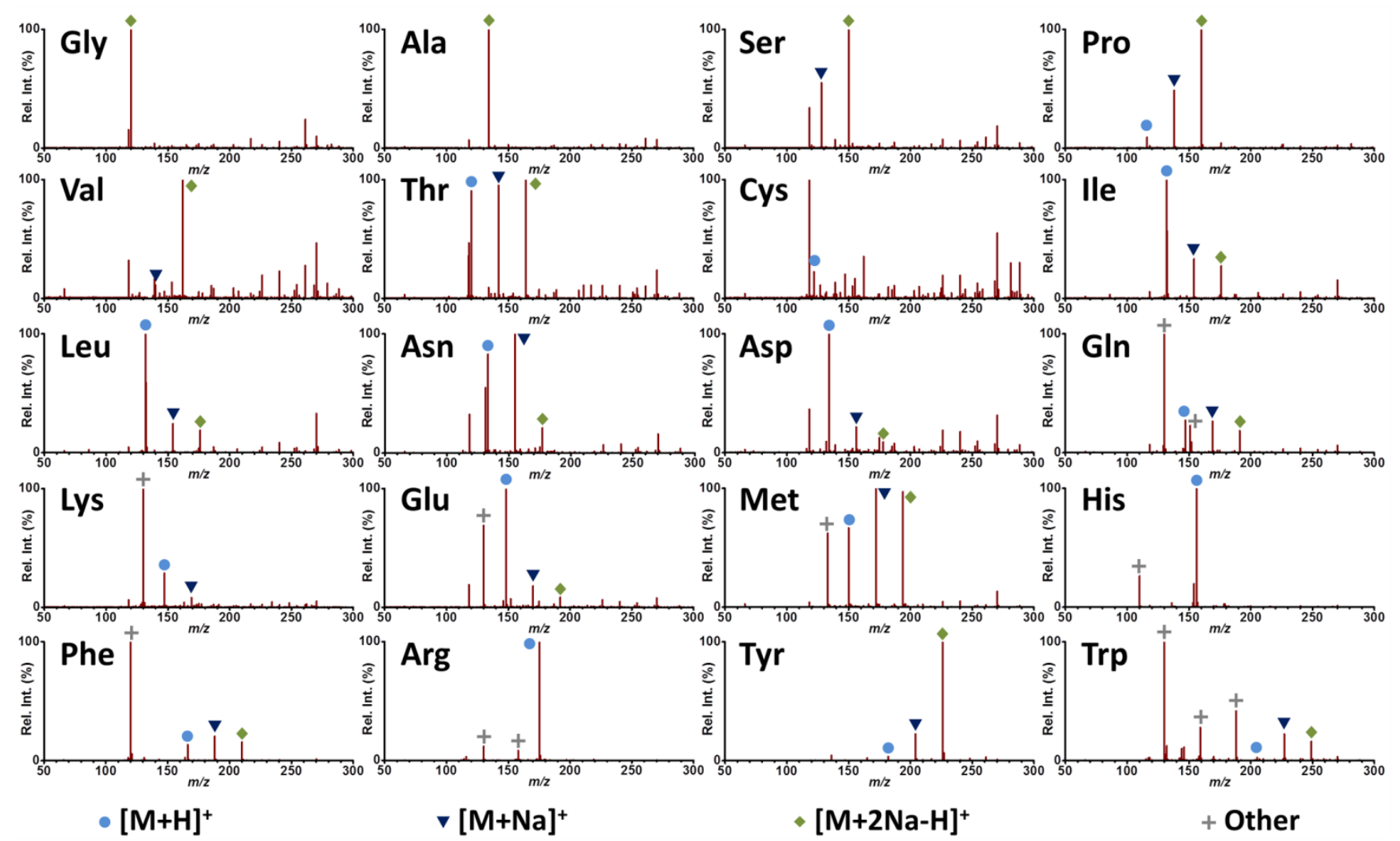

Figure S3. Positive ion mode NAPA-LDI-MS spectra of L-amino acid standards. 


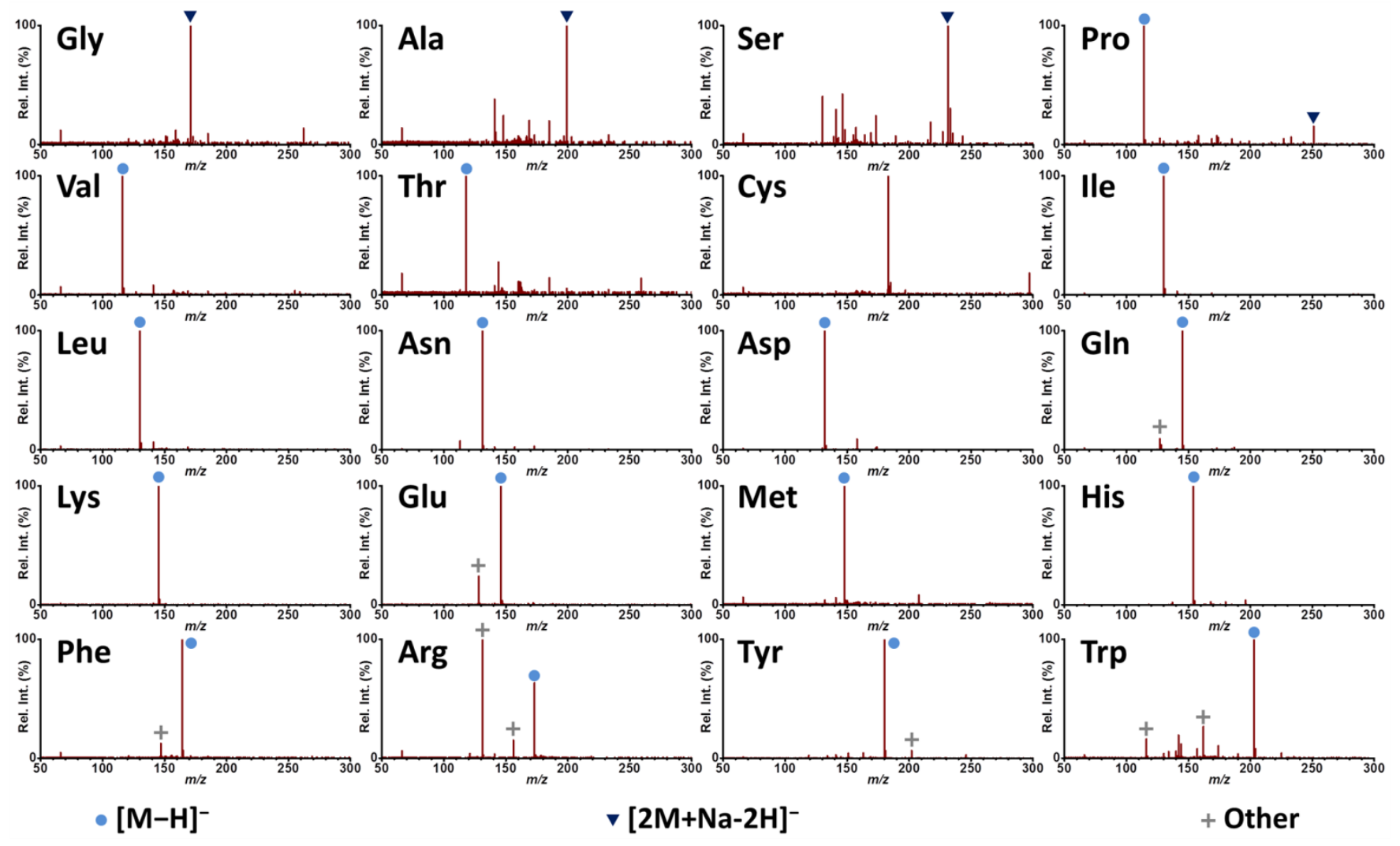

Figure S4. Negative ion mode NAPA-LDI-MS spectra of L-amino acids standards. 

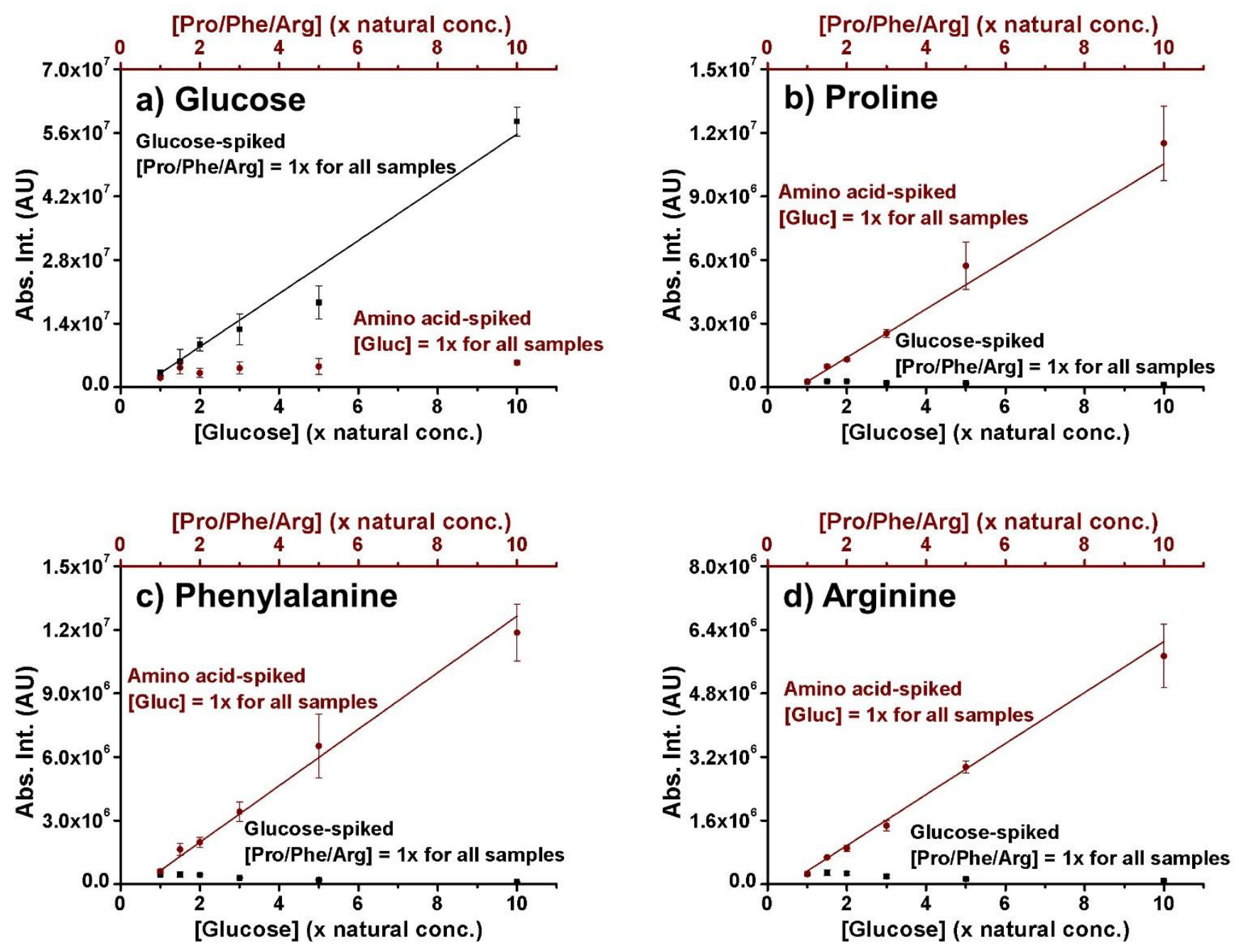

Figure S5. Summed absolute ion intensities for (a) glucose, (b) proline, (c) phenylalanine, and (d) arginine ions as a function of serum spike concentration. Glucose signal includes $[\mathrm{M}+\mathrm{Na}]^{+}$and $[\mathrm{M}+\mathrm{K}]^{+}$species and amino acid signals include $[\mathrm{M}+2 \mathrm{Na}-\mathrm{H}]^{+},[\mathrm{M}+\mathrm{Na}+\mathrm{K}-\mathrm{H}]^{+}$, and $[\mathrm{M}+2 \mathrm{Na}-\mathrm{H}]^{+}$species. Glucose-spiked standards were spiked with glucose in water, while amino acid-spiked standards were spiked with a mixture of proline, phenylalanine, and arginine in water. $R^{2}$ coefficients are 0.97 for glucose in glucose-spiked serum and $>0.99$ for the three amino acids in amino-acid spiked serum. 


\begin{tabular}{|c|c|c|c|c|}
\hline Amino acid & $m / z$ & ID & Composition & Error (mDa) \\
\hline Glycine & 120.0032 & {$[\mathrm{M}+2 \mathrm{Na}-\mathrm{H}]^{+}$} & $\mathrm{C}_{2} \mathrm{H}_{4} \mathrm{O}_{2} \mathrm{NNa}_{2}$ & 0.1 \\
\hline Alanine & 134.0192 & {$[\mathrm{M}+2 \mathrm{Na}-\mathrm{H}]^{+}$} & $\mathrm{C}_{3} \mathrm{H}_{6} \mathrm{O}_{2} \mathrm{NNa}_{2}$ & 0.3 \\
\hline \multirow[t]{2}{*}{ Serine } & 128.0320 & {$[\mathrm{M}+\mathrm{Na}]^{+}$} & $\mathrm{C}_{3} \mathrm{H}_{7} \mathrm{O}_{3} \mathrm{NNa}$ & 0.2 \\
\hline & 150.0139 & {$[\mathrm{M}+2 \mathrm{Na}-\mathrm{H}]^{+}$} & $\mathrm{C}_{3} \mathrm{H}_{6} \mathrm{O}_{3} \mathrm{NNa}_{2}$ & 0.1 \\
\hline \multirow[t]{3}{*}{ Proline } & 116.0707 & {$[\mathrm{M}+\mathrm{H}]^{+}$} & $\mathrm{C}_{5} \mathrm{H}_{10} \mathrm{O}_{2} \mathrm{~N}$ & 0.1 \\
\hline & 138.0528 & {$[\mathrm{M}+\mathrm{Na}]^{+}$} & $\mathrm{C}_{5} \mathrm{H}_{9} \mathrm{O}_{2} \mathrm{NNa}$ & 0.3 \\
\hline & 160.0345 & {$[\mathrm{M}+2 \mathrm{Na}-\mathrm{H}]^{+}$} & $\mathrm{C}_{5} \mathrm{H}_{8} \mathrm{O}_{2} \mathrm{NNa}_{2}$ & 0.0 \\
\hline \multirow[t]{2}{*}{ Valine } & 140.0685 & {$[\mathrm{M}+\mathrm{Na}]^{+}$} & $\mathrm{C}_{5} \mathrm{H}_{11} \mathrm{O}_{2} \mathrm{NNa}$ & 0.3 \\
\hline & 162.0506 & {$[\mathrm{M}+2 \mathrm{Na}-\mathrm{H}]^{+}$} & $\mathrm{C}_{5} \mathrm{H}_{10} \mathrm{O}_{2} \mathrm{NNa}_{2}$ & 0.5 \\
\hline \multirow[t]{3}{*}{ Threonine } & 120.0656 & {$[\mathrm{M}+\mathrm{H}]^{+}$} & $\mathrm{C}_{4} \mathrm{H}_{10} \mathrm{O}_{3} \mathrm{~N}$ & 0.0 \\
\hline & 142.0477 & {$[\mathrm{M}+\mathrm{Na}]^{+}$} & $\mathrm{C}_{4} \mathrm{H}_{9} \mathrm{O}_{3} \mathrm{NNa}$ & 0.3 \\
\hline & 164.0296 & {$[\mathrm{M}+2 \mathrm{Na}-\mathrm{H}]^{+}$} & $\mathrm{C}_{4} \mathrm{H}_{8} \mathrm{O}_{3} \mathrm{NNa}_{2}$ & 0.2 \\
\hline Cysteine & 122.0272 & {$[\mathrm{M}+\mathrm{H}]^{+}$} & $\mathrm{C}_{3} \mathrm{H}_{8} \mathrm{O}_{2} \mathrm{NS}$ & 0.2 \\
\hline \multirow[t]{3}{*}{ Isoleucine } & 132.1023 & {$[\mathrm{M}+\mathrm{H}]^{+}$} & $\mathrm{C}_{6} \mathrm{H}_{14} \mathrm{O}_{2} \mathrm{~N}$ & 0.4 \\
\hline & 154.0842 & {$[\mathrm{M}+\mathrm{Na}]^{+}$} & $\mathrm{C}_{6} \mathrm{H}_{13} \mathrm{O}_{2} \mathrm{NNa}$ & 0.3 \\
\hline & 176.0662 & {$[\mathrm{M}+2 \mathrm{Na}-\mathrm{H}]^{+}$} & $\mathrm{C}_{6} \mathrm{H}_{12} \mathrm{O}_{2} \mathrm{NNa}_{2}$ & 0.4 \\
\hline \multirow[t]{3}{*}{ Leucine } & 132.1023 & {$[\mathrm{M}+\mathrm{H}]^{+}$} & $\mathrm{C}_{6} \mathrm{H}_{14} \mathrm{O}_{2} \mathrm{~N}$ & 0.4 \\
\hline & 154.0842 & {$[\mathrm{M}+\mathrm{Na}]^{+}$} & $\mathrm{C}_{6} \mathrm{H}_{13} \mathrm{O}_{2} \mathrm{NNa}$ & 0.4 \\
\hline & 176.0662 & {$[\mathrm{M}+2 \mathrm{Na}-\mathrm{H}]^{+}$} & $\mathrm{C}_{6} \mathrm{H}_{12} \mathrm{O}_{2} \mathrm{NNa}_{2}$ & 0.4 \\
\hline \multirow[t]{3}{*}{ Asparagine } & 133.0612 & {$[\mathrm{M}+\mathrm{H}]^{+}$} & $\mathrm{C}_{4} \mathrm{H}_{9} \mathrm{O}_{3} \mathrm{~N}_{2}$ & 0.5 \\
\hline & 155.0431 & {$[\mathrm{M}+\mathrm{Na}]^{+}$} & $\mathrm{C}_{4} \mathrm{H}_{8} \mathrm{O}_{3} \mathrm{~N}_{2} \mathrm{Na}$ & 0.3 \\
\hline & 177.0252 & {$[\mathrm{M}+2 \mathrm{Na}-\mathrm{H}]^{+}$} & $\mathrm{C}_{4} \mathrm{H}_{7} \mathrm{O}_{3} \mathrm{~N}_{2} \mathrm{Na}_{2}$ & 0.5 \\
\hline \multirow[t]{3}{*}{ Aspartic acid } & 134.0452 & {$[\mathrm{M}+\mathrm{H}]^{+}$} & $\mathrm{C}_{4} \mathrm{H}_{8} \mathrm{O}_{4} \mathrm{~N}$ & 0.4 \\
\hline & 156.0271 & {$[\mathrm{M}+\mathrm{Na}]^{+}$} & $\mathrm{C}_{4} \mathrm{H}_{7} \mathrm{O}_{4} \mathrm{NNa}$ & 0.4 \\
\hline & 178.0091 & {$[\mathrm{M}+2 \mathrm{Na}-\mathrm{H}]^{+}$} & $\mathrm{C}_{4} \mathrm{H}_{6} \mathrm{O}_{4} \mathrm{NNa}_{2}$ & 0.4 \\
\hline \multirow[t]{5}{*}{ Glutamine } & 130.0503 & {$\left[\mathrm{M}+\mathrm{H}-\mathrm{NH}_{3}\right]^{+}$} & $\mathrm{C}_{5} \mathrm{H}_{8} \mathrm{O}_{3} \mathrm{~N}$ & 0.4 \\
\hline & 147.0768 & {$[\mathrm{M}+\mathrm{H}]^{+}$} & $\mathrm{C}_{5} \mathrm{H}_{11} \mathrm{O}_{3} \mathrm{~N}_{2}$ & 0.4 \\
\hline & 151.0482 & {$\left[\mathrm{M}+\mathrm{Na}-\mathrm{H}_{2} \mathrm{O}\right]^{+}$} & $\mathrm{C}_{5} \mathrm{H}_{8} \mathrm{O}_{2} \mathrm{~N}_{2} \mathrm{Na}$ & 0.4 \\
\hline & 169.0589 & {$[\mathrm{M}+\mathrm{Na}]^{+}$} & $\mathrm{C}_{5} \mathrm{H}_{10} \mathrm{O}_{3} \mathrm{~N}_{2} \mathrm{Na}$ & 0.5 \\
\hline & 191.0408 & {$[\mathrm{M}+2 \mathrm{Na}-\mathrm{H}]^{+}$} & $\mathrm{C}_{5} \mathrm{H}_{9} \mathrm{O}_{3} \mathrm{~N}_{2} \mathrm{Na}_{2}$ & 0.5 \\
\hline \multirow[t]{3}{*}{ Lysine } & 130.0866 & {$\left[\mathrm{M}+\mathrm{H}-\mathrm{NH}_{3}\right]^{+}$} & $\mathrm{C}_{6} \mathrm{H}_{12} \mathrm{O}_{2} \mathrm{~N}$ & 0.4 \\
\hline & 147.1132 & {$[\mathrm{M}+\mathrm{H}]^{+}$} & $\mathrm{C}_{6} \mathrm{H}_{15} \mathrm{O}_{2} \mathrm{~N}_{2}$ & 0.4 \\
\hline & 169.0953 & {$[\mathrm{M}+\mathrm{Na}]^{+}$} & $\mathrm{C}_{6} \mathrm{H}_{14} \mathrm{O}_{2} \mathrm{~N}_{2} \mathrm{Na}$ & 0.6 \\
\hline \multirow[t]{4}{*}{ Glutamic acid } & 130.0503 & {$\left[\mathrm{M}+\mathrm{H}-\mathrm{H}_{2} \mathrm{O}\right]^{+}$} & $\mathrm{C}_{5} \mathrm{H}_{8} \mathrm{O}_{3} \mathrm{~N}$ & 0.4 \\
\hline & 148.0608 & {$[\mathrm{M}+\mathrm{H}]^{+}$} & $\mathrm{C}_{5} \mathrm{H}_{10} \mathrm{O}_{4} \mathrm{~N}$ & 0.3 \\
\hline & 170.0428 & {$[\mathrm{M}+\mathrm{Na}]^{+}$} & $\mathrm{C}_{5} \mathrm{H}_{9} \mathrm{O}_{4} \mathrm{NNa}$ & 0.5 \\
\hline & 192.0249 & {$[\mathrm{M}+2 \mathrm{Na}-\mathrm{H}]^{+}$} & $\mathrm{C}_{5} \mathrm{H}_{8} \mathrm{O}_{4} \mathrm{NNa}_{2}$ & 0.6 \\
\hline
\end{tabular}




\begin{tabular}{|c|c|c|c|c|}
\hline \multirow[t]{4}{*}{ Methionine } & 133.0323 & {$\left[\mathrm{M}+\mathrm{H}-\mathrm{NH}_{3}\right]^{+}$} & $\mathrm{C}_{5} \mathrm{H}_{9} \mathrm{O}_{2} \mathrm{~S}$ & 0.5 \\
\hline & 150.0587 & {$[\mathrm{M}+\mathrm{H}]^{+}$} & $\mathrm{C}_{5} \mathrm{H}_{12} \mathrm{O}_{2} \mathrm{NS}$ & 0.4 \\
\hline & 172.0407 & {$[\mathrm{M}+\mathrm{Na}]^{+}$} & $\mathrm{C}_{5} \mathrm{H}_{11} \mathrm{O}_{2} \mathrm{NNaS}$ & 0.4 \\
\hline & 194.0227 & {$[\mathrm{M}+2 \mathrm{Na}-\mathrm{H}]^{+}$} & $\mathrm{C}_{5} \mathrm{H}_{10} \mathrm{O}_{2} \mathrm{NNa}_{2} \mathrm{~S}$ & 0.5 \\
\hline \multirow[t]{2}{*}{ Histidine } & 110.0714 & {$\left[\mathrm{M}+\mathrm{H}-\mathrm{H}_{2} \mathrm{CO}_{2}\right]^{+}$} & $\mathrm{C}_{5} \mathrm{H}_{8} \mathrm{~N}_{3}$ & 0.1 \\
\hline & 156.0770 & {$[\mathrm{M}+\mathrm{H}]^{+}$} & $\mathrm{C}_{6} \mathrm{H}_{10} \mathrm{O}_{2} \mathrm{~N}_{3}$ & 0.2 \\
\hline \multirow[t]{4}{*}{ Phenylalanine } & 120.0809 & {$\left[\mathrm{M}+\mathrm{H}-\mathrm{H}_{2} \mathrm{CO}_{2}\right]^{+}$} & $\mathrm{C}_{8} \mathrm{H}_{10} \mathrm{~N}$ & 0.2 \\
\hline & 166.0866 & {$[\mathrm{M}+\mathrm{H}]^{+}$} & $\mathrm{C}_{9} \mathrm{H}_{12} \mathrm{O}_{2} \mathrm{~N}$ & 0.4 \\
\hline & 188.0687 & {$[\mathrm{M}+\mathrm{Na}]^{+}$} & $\mathrm{C}_{9} \mathrm{H}_{11} \mathrm{O}_{2} \mathrm{NNa}$ & 0.5 \\
\hline & 210.0506 & {$[\mathrm{M}+2 \mathrm{Na}-\mathrm{H}]^{+}$} & $\mathrm{C}_{9} \mathrm{H}_{10} \mathrm{O}_{2} \mathrm{NNa}_{2}$ & 0.5 \\
\hline \multirow[t]{3}{*}{ Arginine } & 130.0978 & {$\left[\mathrm{M}+\mathrm{H}-\mathrm{CH}_{3} \mathrm{NO}\right]^{+}$} & $\mathrm{C}_{5} \mathrm{H}_{12} \mathrm{ON}_{3}$ & 0.3 \\
\hline & 158.0927 & {$\left[\mathrm{M}+\mathrm{H}-\mathrm{NH}_{3}\right]^{+}$} & $\mathrm{C}_{6} \mathrm{H}_{12} \mathrm{O}_{2} \mathrm{~N}_{3}$ & 0.3 \\
\hline & 175.1193 & {$[\mathrm{M}+\mathrm{H}]^{+}$} & $\mathrm{C}_{6} \mathrm{H}_{15} \mathrm{O}_{2} \mathrm{~N}_{4}$ & 0.4 \\
\hline \multirow[t]{3}{*}{ Tyrosine } & 182.0817 & {$[\mathrm{M}+\mathrm{H}]^{+}$} & $\mathrm{C}_{9} \mathrm{H}_{12} \mathrm{O}_{3} \mathrm{~N}$ & 0.6 \\
\hline & 204.0635 & {$[\mathrm{M}+\mathrm{Na}]^{+}$} & $\mathrm{C}_{9} \mathrm{H}_{11} \mathrm{O}_{3} \mathrm{NNa}$ & 0.4 \\
\hline & 226.0455 & {$[\mathrm{M}+2 \mathrm{Na}-\mathrm{H}]^{+}$} & $\mathrm{C}_{9} \mathrm{H}_{10} \mathrm{O}_{3} \mathrm{NNa}_{2}$ & 0.4 \\
\hline \multirow[t]{6}{*}{ Tryptophan } & 130.0654 & {$\left[\mathrm{M}+\mathrm{H}-\mathrm{C}_{2} \mathrm{H}_{5} \mathrm{NO}_{2}\right]^{+}$} & $\mathrm{C}_{9} \mathrm{H}_{8} \mathrm{~N}$ & 0.3 \\
\hline & 159.0919 & {$\left[\mathrm{M}+\mathrm{H}-\mathrm{CH}_{2} \mathrm{O}_{2}\right]^{+}$} & $\mathrm{C}_{10} \mathrm{H}_{11} \mathrm{~N}_{2}$ & 0.2 \\
\hline & 188.0710 & {$\left[\mathrm{M}+\mathrm{H}-\mathrm{NH}_{3}\right]^{+}$} & $\mathrm{C}_{11} \mathrm{H}_{10} \mathrm{O}_{2} \mathrm{~N}$ & 0.4 \\
\hline & 205.0976 & {$[\mathrm{M}+\mathrm{H}]^{+}$} & $\mathrm{C}_{11} \mathrm{H}_{13} \mathrm{O}_{2} \mathrm{~N}_{2}$ & 0.4 \\
\hline & 227.0795 & {$[\mathrm{M}+\mathrm{Na}]^{+}$} & $\mathrm{C}_{11} \mathrm{H}_{12} \mathrm{O}_{2} \mathrm{~N}_{2} \mathrm{Na}$ & 0.4 \\
\hline & 249.0615 & {$[\mathrm{M}+2 \mathrm{Na}-\mathrm{H}]^{+}$} & $\mathrm{C}_{11} \mathrm{H}_{11} \mathrm{O}_{2} \mathrm{~N}_{2} \mathrm{Na}_{2}$ & 0.4 \\
\hline
\end{tabular}




\begin{tabular}{|c|c|c|c|c|}
\hline Amino acid & $m / z$ & ID & Composition & Error (mDa) \\
\hline Glycine & 171.0386 & {$[2 \mathrm{M}+\mathrm{Na}-2 \mathrm{H}]^{-}$} & $\mathrm{C}_{4} \mathrm{H}_{8} \mathrm{O}_{4} \mathrm{~N}_{2} \mathrm{Na}$ & -0.1 \\
\hline Alanine & 199.0705 & {$[2 \mathrm{M}+\mathrm{Na}-2 \mathrm{H}]^{-}$} & $\mathrm{C}_{6} \mathrm{H}_{12} \mathrm{O}_{4} \mathrm{~N}_{2} \mathrm{Na}$ & 0.5 \\
\hline Serine & 231.0613 & {$[2 \mathrm{M}+\mathrm{Na}-2 \mathrm{H}]^{-}$} & $\mathrm{C}_{6} \mathrm{H}_{12} \mathrm{O}_{6} \mathrm{~N}_{2} \mathrm{Na}$ & 1.4 \\
\hline \multirow[t]{2}{*}{ Proline } & 114.0560 & {$[\mathrm{M}-\mathrm{H}]^{-}$} & $\mathrm{C}_{5} \mathrm{H}_{8} \mathrm{O}_{2} \mathrm{~N}$ & -0.1 \\
\hline & 251.1033 & {$[2 \mathrm{M}+\mathrm{Na}-2 \mathrm{H}]^{-}$} & $\mathrm{C}_{10} \mathrm{H}_{16} \mathrm{O}_{4} \mathrm{~N}_{2} \mathrm{Na}$ & 2.0 \\
\hline Valine & 116.0714 & {$[\mathrm{M}-\mathrm{H}]^{-}$} & $\mathrm{C}_{5} \mathrm{H}_{10} \mathrm{O}_{2} \mathrm{~N}$ & -0.3 \\
\hline Threonine & 118.0507 & {$[\mathrm{M}-\mathrm{H}]^{-}$} & $\mathrm{C}_{4} \mathrm{H}_{8} \mathrm{O}_{3} \mathrm{~N}$ & -0.2 \\
\hline Cysteine & - & - & - & - \\
\hline Isoleucine & 130.0871 & {$[\mathrm{M}-\mathrm{H}]^{-}$} & $\mathrm{C}_{6} \mathrm{H}_{12} \mathrm{O}_{2} \mathrm{~N}$ & -0.2 \\
\hline Leucine & 130.0871 & {$[\mathrm{M}-\mathrm{H}]^{-}$} & $\mathrm{C}_{6} \mathrm{H}_{12} \mathrm{O}_{2} \mathrm{~N}$ & -0.2 \\
\hline Asparagine & 131.0461 & {$[\mathrm{M}-\mathrm{H}]^{-}$} & $\mathrm{C}_{4} \mathrm{H}_{7} \mathrm{O}_{3} \mathrm{~N}_{2}$ & -0.1 \\
\hline Aspartic acid & 132.0303 & {$[\mathrm{M}-\mathrm{H}]^{-}$} & $\mathrm{C}_{4} \mathrm{H}_{6} \mathrm{O}_{4} \mathrm{~N}$ & 0.1 \\
\hline \multirow[t]{2}{*}{ Glutamine } & 127.0507 & {$[\mathrm{M}-\mathrm{H}]^{-}$} & $\mathrm{C}_{5} \mathrm{H}_{7} \mathrm{O}_{2} \mathrm{~N}_{2}$ & 0.0 \\
\hline & 145.0619 & {$[\mathrm{M}-\mathrm{H}]^{-}$} & $\mathrm{C}_{5} \mathrm{H}_{9} \mathrm{O}_{3} \mathrm{~N}_{2}$ & 0.0 \\
\hline Lysine & 145.0983 & {$[\mathrm{M}-\mathrm{H}]^{-}$} & $\mathrm{C}_{6} \mathrm{H}_{13} \mathrm{O}_{2} \mathrm{~N}_{2}$ & 0.0 \\
\hline \multirow[t]{2}{*}{ Glutamic acid } & 128.0351 & {$[\mathrm{M}-\mathrm{H}]^{-}$} & $\mathrm{C}_{5} \mathrm{H}_{6} \mathrm{O}_{3} \mathrm{~N}$ & -0.2 \\
\hline & 146.0460 & {$[\mathrm{M}-\mathrm{H}]^{-}$} & $\mathrm{C}_{5} \mathrm{H}_{8} \mathrm{O}_{4} \mathrm{~N}$ & 0.1 \\
\hline Methionine & 148.0437 & {$[\mathrm{M}-\mathrm{H}]^{-}$} & $\mathrm{C}_{5} \mathrm{H}_{10} \mathrm{O}_{2} \mathrm{NS}$ & -0.1 \\
\hline Histidine & 154.0625 & {$[\mathrm{M}-\mathrm{H}]^{-}$} & $\mathrm{C}_{6} \mathrm{H}_{8} \mathrm{O}_{2} \mathrm{~N}_{3}$ & 0.3 \\
\hline \multirow[t]{2}{*}{ Phenylalanine } & 147.0452 & {$\left[\mathrm{M}-\mathrm{H}-\mathrm{NH}_{3}\right]^{-}$} & $\mathrm{C}_{9} \mathrm{H}_{7} \mathrm{O}_{2}$ & 0.0 \\
\hline & 164.0714 & {$[\mathrm{M}-\mathrm{H}]^{-}$} & $\mathrm{C}_{9} \mathrm{H}_{10} \mathrm{O}_{2} \mathrm{~N}$ & -0.3 \\
\hline \multirow[t]{3}{*}{ Arginine } & 131.0819 & {$\left[\mathrm{M}-\mathrm{H}-\mathrm{CH}_{2} \mathrm{~N}_{2}\right]^{-}$} & $\mathrm{C}_{5} \mathrm{H}_{11} \mathrm{O}_{2} \mathrm{~N}_{2}$ & -0.3 \\
\hline & 156.0776 & {$\left[\mathrm{M}-\mathrm{H}-\mathrm{NH}_{3}\right]^{-}$} & $\mathrm{C}_{6} \mathrm{H}_{10} \mathrm{O}_{2} \mathrm{~N}_{3}$ & 0.5 \\
\hline & 173.1050 & {$[\mathrm{M}-\mathrm{H}]^{-}$} & $\mathrm{C}_{6} \mathrm{H}_{13} \mathrm{O}_{2} \mathrm{~N}_{4}$ & 0.0 \\
\hline \multirow[t]{2}{*}{ Tyrosine } & 180.0663 & {$[\mathrm{M}-\mathrm{H}]^{-}$} & $\mathrm{C}_{9} \mathrm{H}_{10} \mathrm{O}_{3} \mathrm{~N}$ & -0.3 \\
\hline & 202.0482 & {$[\mathrm{M}-2 \mathrm{H}+\mathrm{Na}]^{-}$} & $\mathrm{C}_{9} \mathrm{H}_{9} \mathrm{O}_{3} \mathrm{NNa}$ & -0.3 \\
\hline \multirow[t]{3}{*}{ Tryptophan } & 116.0512 & {$\left[\mathrm{M}-\mathrm{H}-\mathrm{C}_{3} \mathrm{H}_{5} \mathrm{NO}_{2}\right]^{-}$} & $\mathrm{C}_{8} \mathrm{H}_{6} \mathrm{~N}$ & 0.6 \\
\hline & 162.0560 & {$\left[\mathrm{M}-\mathrm{H}-\mathrm{C}_{2} \mathrm{H}_{3} \mathrm{~N}\right]^{-}$} & $\mathrm{C}_{9} \mathrm{H}_{8} \mathrm{O}_{2} \mathrm{~N}$ & -0.1 \\
\hline & 203.0828 & {$[\mathrm{M}-\mathrm{H}]^{-}$} & $\mathrm{C}_{11} \mathrm{H}_{11} \mathrm{O}_{2} \mathrm{~N}_{2}$ & 0.2 \\
\hline
\end{tabular}




\begin{tabular}{|c|c|c|c|}
\hline$m / z$ & Assignment & Ion & Error (mDa) \\
\hline 118.0858 & Betaine & {$[\mathrm{M}+\mathrm{H}]^{+}$} & -0.5 \\
\hline 120.9658 & Phosphoric acid & {$[\mathrm{M}+\mathrm{Na}]^{+}$} & -0.3 \\
\hline 122.9238 & Formic acid & {$[\mathrm{M}+2 \mathrm{~K}-\mathrm{H}]^{+}$} & -0.7 \\
\hline 132.0474 & Methionine & {$\left[\mathrm{M}+\mathrm{H}-\mathrm{H}_{2} \mathrm{O}\right]^{+}$} & -0.4 \\
\hline 132.987 & Pyruvic acid & {$[\mathrm{M}+2 \mathrm{Na}-\mathrm{H}]^{+}$} & -0.2 \\
\hline 134.0186 & Alanine & {$[\mathrm{M}+2 \mathrm{Na}-\mathrm{H}]^{+}$} & -0.2 \\
\hline 135.0026 & Lactic acid & {$[\mathrm{M}+2 \mathrm{Na}-\mathrm{H}]^{+}$} & -0.3 \\
\hline 136.0478 & Creatinine & {$[\mathrm{M}+\mathrm{Na}]^{+}$} & -0.3 \\
\hline 136.9399 & Phosphoric acid & {$[\mathrm{M}+\mathrm{K}]^{+}$} & -0.2 \\
\hline 142.9477 & Phosphoric acid & {$[\mathrm{M}+2 \mathrm{Na}-\mathrm{H}]^{+}$} & -0.4 \\
\hline 143.0312 & 3,4-dihydroxybutyric acid & {$[\mathrm{M}+\mathrm{Na}]^{+}$} & -0.3 \\
\hline 147.0026 & Acetoacetic acid & {$[\mathrm{M}+2 \mathrm{Na}-\mathrm{H}]^{+}$} & -0.3 \\
\hline 148.9608 & Pyruvic acid & {$[\mathrm{M}+\mathrm{Na}+\mathrm{K}-\mathrm{H}]^{+}$} & -0.3 \\
\hline 149.0181 & 3-hydroxybutanoic acid & {$[\mathrm{M}+2 \mathrm{Na}-\mathrm{H}]^{+}$} & -0.4 \\
\hline 152.0217 & Creatinine & {$[\mathrm{M}+\mathrm{K}]^{+}$} & -0.4 \\
\hline 158.0296 & Creatinine & {$[\mathrm{M}+2 \mathrm{Na}-\mathrm{H}]^{+}$} & -0.5 \\
\hline 160.0341 & Proline & {$[\mathrm{M}+2 \mathrm{Na}-\mathrm{H}]^{+}$} & -0.4 \\
\hline 162.0134 & N-Acetylglycine & {$[\mathrm{M}+2 \mathrm{Na}-\mathrm{H}]^{+}$} & -0.4 \\
\hline 162.0497 & Valine & {$[\mathrm{M}+2 \mathrm{Na}-\mathrm{H}]^{+}$} & -0.4 \\
\hline 164.9347 & Pyruvic acid & {$[\mathrm{M}+2 \mathrm{~K}-\mathrm{H}]^{+}$} & -0.4 \\
\hline 165.9664 & Alanine & {$[\mathrm{M}+2 \mathrm{~K}-\mathrm{H}]^{+}$} & -0.3 \\
\hline 166.9505 & Lactic acid & {$[\mathrm{M}+2 \mathrm{~K}-\mathrm{H}]^{+}$} & -0.2 \\
\hline 174.0134 & Pyroglutamic acid & {$[\mathrm{M}+2 \mathrm{Na}-\mathrm{H}]^{+}$} & -0.4 \\
\hline 174.8956 & Phosphoric acid & {$[\mathrm{M}+2 \mathrm{~K}-\mathrm{H}]^{+}$} & -0.3 \\
\hline 174.9976 & Citraconic acid & {$[\mathrm{M}+2 \mathrm{Na}-\mathrm{H}]^{+}$} & -0.2 \\
\hline 175.024 & Citric acid & {$\left[\mathrm{M}+\mathrm{H}-\mathrm{H}_{2} \mathrm{O}\right]^{+}$} & 0.3 \\
\hline 176.029 & Trans-4-hydroxyproline & {$[\mathrm{M}+2 \mathrm{Na}-\mathrm{H}]^{+}$} & -0.4 \\
\hline 176.0401 & Creatine & {$[\mathrm{M}+2 \mathrm{Na}-\mathrm{H}]^{+}$} & -0.5 \\
\hline 176.0654 & Leucine/isoleucine & {$[\mathrm{M}+2 \mathrm{Na}-\mathrm{H}]^{+}$} & -0.4 \\
\hline 177.0616 & Ornithine & {$[\mathrm{M}+2 \mathrm{Na}-\mathrm{H}]^{+}$} & 0.6 \\
\hline 183.0026 & Salicylic acid & {$[\mathrm{M}+2 \mathrm{Na}-\mathrm{H}]^{+}$} & -0.3 \\
\hline 191.0399 & Glutamine & {$[\mathrm{M}+2 \mathrm{Na}-\mathrm{H}]^{+}$} & -0.4 \\
\hline 191.077 & Lysine & {$[\mathrm{M}+2 \mathrm{Na}-\mathrm{H}]^{+}$} & 0.3 \\
\hline 191.982 & Proline & {$[\mathrm{M}+2 \mathrm{~K}-\mathrm{H}]^{+}$} & -0.4 \\
\hline 193.9978 & Valine & {$[\mathrm{M}+2 \mathrm{~K}-\mathrm{H}]^{+}$} & -0.2 \\
\hline 200.0404 & Histidine & {$[\mathrm{M}+2 \mathrm{Na}-\mathrm{H}]^{+}$} & -0.2 \\
\hline 203.0522 & Hexose & {$[\mathrm{M}+\mathrm{Na}]^{+}$} & -0.4 \\
\hline 205.9613 & Pyroglutamic acid & {$[\mathrm{M}+2 \mathrm{~K}-\mathrm{H}]^{+}$} & -0.3 \\
\hline 207.9769 & Trans-4-hydroxyproline & {$[\mathrm{M}+2 \mathrm{~K}-\mathrm{H}]^{+}$} & -0.4 \\
\hline
\end{tabular}


208.0133

210.0497

219.0261

219.0824

222.9878

226.0446

233.0866

241.9976

249.0606

251.0305

265.0345

273.1794

279.2285

299.1949

301.2109

303.2291

305.245

325.2107

327.2264

329.2421

387.0912

Leucine/isoleucine
Phenylalanine
Hexose
Arginine
Glutamine
Tyrosine
N-alpha-acetyl-L-lysine
Phenylalanine
Tryptophan
Arginine

$\mathrm{N}$-alpha-acetyl-L-lysine

Myristic acid

Palmitic acid

Palmitelaidic acid

Palmitic acid

Linoleic acid

Oleic acid

Linoleic acid

Oleic acid

Stearic acid

Maltose

$\begin{array}{cc}{[\mathrm{M}+2 \mathrm{~K}-\mathrm{H}]^{+}} & -0.4 \\ {[\mathrm{M}+2 \mathrm{Na}-\mathrm{H}]^{+}} & -0.4 \\ {[\mathrm{M}+\mathrm{K}]^{+}} & -0.5 \\ {[\mathrm{M}+2 \mathrm{Na}-\mathrm{H}]^{+}} & -0.4 \\ {[\mathrm{M}+2 \mathrm{~K}-\mathrm{H}]^{+}} & -0.4 \\ {[\mathrm{M}+2 \mathrm{Na}-\mathrm{H}]^{+}} & -0.5 \\ {[\mathrm{M}+2 \mathrm{Na}-\mathrm{H}]^{+}} & -0.7 \\ {[\mathrm{M}+2 \mathrm{~K}-\mathrm{H}]^{+}} & -0.4 \\ {[\mathrm{M}+2 \mathrm{Na}-\mathrm{H}]^{+}} & -0.4 \\ {[\mathrm{M}+2 \mathrm{~K}-\mathrm{H}]^{+}} & -0.2 \\ {[\mathrm{M}+2 \mathrm{~K}-\mathrm{H}]^{+}} & -0.6 \\ {[\mathrm{M}+2 \mathrm{Na}-\mathrm{H}]^{+}} & -0.7 \\ {[\mathrm{M}+\mathrm{Na}]^{+}} & -1.0 \\ {[\mathrm{M}+2 \mathrm{Na}-\mathrm{H}]^{+}} & -0.8 \\ {[\mathrm{M}+2 \mathrm{Na}-\mathrm{H}]^{+}} & -0.5 \\ {[\mathrm{M}+\mathrm{Na}]^{+}} & -0.4 \\ {[\mathrm{M}+\mathrm{Na}]^{+}} & -0.1 \\ {[\mathrm{M}+2 \mathrm{Na}-\mathrm{H}]^{+}} & -0.7 \\ {[\mathrm{M}+2 \mathrm{Na}-\mathrm{H}]^{+}} & -0.7 \\ {[\mathrm{M}+2 \mathrm{Na}-\mathrm{H}]^{+}} & -0.6 \\ {[\mathrm{M}+2 \mathrm{Na}-\mathrm{H}]^{+}} & 3.8\end{array}$




\begin{tabular}{|c|c|c|c|}
\hline$m / z$ & Assignment & Ion & Error (mDa) \\
\hline 121.0297 & Benzoic acid & {$[\mathrm{M}-\mathrm{H}]^{-}$} & 0.2 \\
\hline 127.0515 & Glutamine & {$\left[\mathrm{M}-\mathrm{H}-\mathrm{H}_{2} \mathrm{O}\right]^{-}$} & 0.2 \\
\hline 127.0879 & Lysine & {$\left[\mathrm{M}-\mathrm{H}-\mathrm{H}_{2} \mathrm{O}\right]^{-}$} & 0.2 \\
\hline 128.0354 & Pyroglutamic acid & {$[\mathrm{M}-\mathrm{H}]^{-}$} & 0.1 \\
\hline 130.0624 & Creatine & {$[\mathrm{M}-\mathrm{H}]^{-}$} & 0.2 \\
\hline 130.0872 & Leucine/isoleucine & {$[\mathrm{M}-\mathrm{H}]^{-}$} & -0.1 \\
\hline 137.0243 & Salicylic acid & {$[\mathrm{M}-\mathrm{H}]^{-}$} & -0.1 \\
\hline 145.0617 & Glutamine & {$[\mathrm{M}-\mathrm{H}]^{-}$} & -0.2 \\
\hline 146.0611 & Phenylalanine & {$\left[\mathrm{M}-\mathrm{H}-\mathrm{H}_{2} \mathrm{O}\right]^{-}$} & -0.0 \\
\hline 150.0561 & Acetaminophen & {$[\mathrm{M}-\mathrm{H}]^{-}$} & 0.1 \\
\hline 154.062 & Histidine & {$[\mathrm{M}-\mathrm{H}]^{-}$} & -0.2 \\
\hline 161.0457 & Hexose & {$\left[\mathrm{M}-\mathrm{H}-\mathrm{H}_{2} \mathrm{O}\right]^{-}$} & 0.2 \\
\hline 162.0562 & Tyrosine & {$\left[\mathrm{M}-\mathrm{H}-\mathrm{H}_{2} \mathrm{O}\right]^{-}$} & 0.2 \\
\hline 164.0715 & Phenylalanine & {$[\mathrm{M}-\mathrm{H}]^{-}$} & -0.2 \\
\hline 168.0426 & Leucine/isoleucine & {$[\mathrm{M}-2 \mathrm{H}+\mathrm{K}]^{-}$} & -0.6 \\
\hline 169.0982 & $\mathrm{~N}$-alpha-acetyl-L-lysine & {$\left[\mathrm{M}-\mathrm{H}-\mathrm{H}_{2} \mathrm{O}\right]^{-}$} & -0.0 \\
\hline 180.067 & Tyrosine & {$[\mathrm{M}-\mathrm{H}]^{-}$} & 0.4 \\
\hline 185.0716 & Tryptophan & {$\left[\mathrm{M}-\mathrm{H}-\mathrm{H}_{2} \mathrm{O}\right]^{-}$} & -0.4 \\
\hline 189.0026 & Uric acid & {$[\mathrm{M}-2 \mathrm{H}+\mathrm{Na}]^{-}$} & -0.4 \\
\hline 202.0501 & Tyrosine & {$[\mathrm{M}-2 \mathrm{H}+\mathrm{Na}]^{-}$} & 1.5 \\
\hline 203.0828 & Tryptophan & {$[\mathrm{M}-\mathrm{H}]^{-}$} & 0.2 \\
\hline 209.0935 & Capric acid & {$[\mathrm{M}-2 \mathrm{H}+\mathrm{K}]^{-}$} & -1.4 \\
\hline 227.2022 & Myristic acid & {$[\mathrm{M}-\mathrm{H}]^{-}$} & 0.5 \\
\hline 237.125 & Dodecanoic acid & {$[\mathrm{M}-2 \mathrm{H}+\mathrm{K}]^{-}$} & -1.2 \\
\hline 253.2176 & Palmitelaidic acid & {$[\mathrm{M}-\mathrm{H}]^{-}$} & 0.3 \\
\hline 255.2334 & Palmitic acid & {$[\mathrm{M}-\mathrm{H}]^{-}$} & 0.5 \\
\hline 269.2495 & Heptadecanoic acid & {$[\mathrm{M}-\mathrm{H}]^{-}$} & 0.9 \\
\hline 279.2328 & Linoleic acid & {$[\mathrm{M}-\mathrm{H}]^{-}$} & -0.2 \\
\hline 281.2482 & Oleic acid & {$[\mathrm{M}-\mathrm{H}]^{-}$} & -0.4 \\
\hline 283.264 & Stearic acid & {$[\mathrm{M}-\mathrm{H}]^{-}$} & -0.3 \\
\hline
\end{tabular}


Table S5. Tentatively assigned ionic species detected in positive ion mode NAPA-LDI-MS analysis of organic phase serum extracts. CPA: cyclophosphatidic acid; LPA: Iysophosphatidic acid; PA: phosphatidic acid; LPC: lysophosphatidylcholine; PC: phosphatidylcholine; LPE: lysophosphatidylethanolamine; PE: phosphatidylethanolamine; LPG: lysophosphatidylglycerol; DG: diacylglycerol; SM: sphingomyelin.

\begin{tabular}{|c|c|c|c|}
\hline$m / z$ & Assignment & Ion & Error (mDa) \\
\hline 258.1093 & Glycerophosphocholine & {$[\mathrm{M}+\mathrm{H}]^{+}$} & -0.8 \\
\hline 301.2104 & Palmitic acid & {$[\mathrm{M}+2 \mathrm{Na}-\mathrm{H}]^{+}$} & -0.9 \\
\hline 367.2832 & Dihydroxyeicosanoic acid & {$[\mathrm{M}+\mathrm{Na}]^{+}$} & 1.3 \\
\hline 369.3505 & Cholesterol & {$\left[\mathrm{M}+\mathrm{H}-\mathrm{H}_{2} \mathrm{O}\right]^{+}$} & -1.1 \\
\hline 415.2205 & CPA $(16: 0)$ & {$[\mathrm{M}+\mathrm{Na}]^{+}$} & -1.5 \\
\hline 419.2543 & CPA $(18: 1)$ & {$[\mathrm{M}+\mathrm{H}]^{+}$} & -1.4 \\
\hline 437.2026 & CPA (16:0) & {$[\mathrm{M}+2 \mathrm{Na}-\mathrm{H}]^{+}$} & -1.3 \\
\hline 439.2199 & CPA (18:2) & {$[\mathrm{M}+\mathrm{Na}]^{+}$} & -2.1 \\
\hline 441.2361 & CPA $(18: 1)$ & {$[\mathrm{M}+\mathrm{Na}]^{+}$} & -1.5 \\
\hline 443.2522 & CPA (18:0) & {$[\mathrm{M}+\mathrm{Na}]^{+}$} & -1.1 \\
\hline 447.2852 & LPA (18:0e) & {$[\mathrm{M}+\mathrm{Na}]^{+}$} & 0.6 \\
\hline 455.2118 & LPA (16:0) & {$[\mathrm{M}+2 \mathrm{Na}-\mathrm{H}]^{+}$} & -2.7 \\
\hline 457.2308 & LPA (18:2) & {$[\mathrm{M}+\mathrm{Na}]^{+}$} & -1.7 \\
\hline 459.2466 & LPA $(18: 1)$ & {$[\mathrm{M}+\mathrm{Na}]^{+}$} & -1.6 \\
\hline 461.2022 & CPA (18:2) & {$[\mathrm{M}+2 \mathrm{Na}-\mathrm{H}]^{+}$} & -1.7 \\
\hline 463.2182 & CPA $(18: 1)$ & {$[\mathrm{M}+2 \mathrm{Na}-\mathrm{H}]^{+}$} & -1.4 \\
\hline 469.2674 & LPA (18:0e) & {$[\mathrm{M}+2 \mathrm{Na}-\mathrm{H}]^{+}$} & 0.9 \\
\hline 483.2465 & LPA $(18: 0)$ & {$[\mathrm{M}+2 \mathrm{Na}-\mathrm{H}]^{+}$} & 0.7 \\
\hline 502.3274 & LPC (P-16:0) & {$[\mathrm{M}+\mathrm{Na}]^{+}$} & 0.6 \\
\hline 506.3598 & LPC (P-18:1) & {$[\mathrm{M}+\mathrm{H}]^{+}$} & -0.7 \\
\hline 510.3538 & LPE (20:0) & {$[\mathrm{M}+\mathrm{H}]^{+}$} & -1.6 \\
\hline 518.3199 & LPC (16:0) & {$[\mathrm{M}+\mathrm{Na}]^{+}$} & -1.9 \\
\hline 524.3693 & LPC (18:0) & {$[\mathrm{M}+\mathrm{H}]^{+}$} & -1.8 \\
\hline 542.3201 & LPC (18:2) & {$[\mathrm{M}+\mathrm{Na}]^{+}$} & -1.6 \\
\hline 544.3357 & LPC (18:1) & {$[\mathrm{M}+\mathrm{Na}]^{+}$} & -1.6 \\
\hline 546.3513 & LPC (18:0) & {$[\mathrm{M}+\mathrm{Na}]^{+}$} & -1.7 \\
\hline 557.2832 & LPG (18:0) & {$[\mathrm{M}+2 \mathrm{Na}-\mathrm{H}]^{+}$} & 0.6 \\
\hline 613.4786 & DG (34:3) & {$[\mathrm{M}+\mathrm{Na}]^{+}$} & -1.6 \\
\hline 637.4786 & DG $(36: 5)$ & {$[\mathrm{M}+\mathrm{Na}]^{+}$} & -1.6 \\
\hline 639.4951 & DG $(36: 4)$ & {$[\mathrm{M}+\mathrm{Na}]^{+}$} & -0.8 \\
\hline 665.5102 & DG (38:5) & {$[\mathrm{M}+\mathrm{Na}]^{+}$} & -1.4 \\
\hline 723.4922 & PA (36:2) & {$[\mathrm{M}+\mathrm{Na}]^{+}$} & -1.3 \\
\hline 725.5548 & SM (d34:1) & {$[\mathrm{M}+\mathrm{Na}]^{+}$} & -2.0 \\
\hline 745.4763 & PA (36:2) & {$[\mathrm{M}+2 \mathrm{Na}-\mathrm{H}]^{+}$} & 0.8 \\
\hline 772.5832 & PE (38:2) & {$[\mathrm{M}+\mathrm{H}]^{+}$} & -1.9 \\
\hline 774.5983 & PE (38:1) & {$[\mathrm{M}+\mathrm{H}]^{+}$} & -2.5 \\
\hline
\end{tabular}


780.5493

782.5653

800.6147

804.5497

806.5654

808.5807

832.5809
PC (34:1)

PC (34:1)

PE (40:2)

PC (34:1)

PC (36:3)

PC (36:2)

PC (38:4)

$\begin{array}{cr}{[\mathrm{M}+\mathrm{Na}]^{+}} & -2.0 \\ {[\mathrm{M}+\mathrm{Na}]^{+}} & -1.8 \\ {[\mathrm{M}+\mathrm{H}]^{+}} & -1.7 \\ {[\mathrm{M}+2 \mathrm{Na}-\mathrm{H}]^{+}} & 0.7 \\ {[\mathrm{M}+\mathrm{Na}]^{+}} & -1.6 \\ {[\mathrm{M}+\mathrm{Na}]^{+}} & -2.0 \\ {[\mathrm{M}+\mathrm{Na}]^{+}} & -1.8\end{array}$

$-2.0$

$-1.7$

0.7

$-1.8$ 


\begin{tabular}{|c|c|c|c|}
\hline$m / z$ & Assignment & Ion & Error (mDa) \\
\hline 209.0800 & Azelaic acid & {$[\mathrm{M}+\mathrm{Na}-2 \mathrm{H}]^{-}$} & 0.4 \\
\hline 211.1344 & Dodecanedioic acid & {$\left[\mathrm{M}-\mathrm{H}-\mathrm{H}_{2} \mathrm{O}\right]^{-}$} & 1.0 \\
\hline 227.2024 & Myristic acid & {$[\mathrm{M}-\mathrm{H}]^{-}$} & 0.7 \\
\hline 241.0122 & Inositol phosphate & {$\left[\mathrm{M}-\mathrm{H}-\mathrm{H}_{2} \mathrm{O}\right]^{-}$} & 0.9 \\
\hline 253.2180 & Palmitelaidic acid & {$[\mathrm{M}-\mathrm{H}]^{-}$} & 0.7 \\
\hline 255.2337 & Palmitic acid & {$[\mathrm{M}-\mathrm{H}]^{-}$} & 0.7 \\
\hline 259.0227 & Inositol phosphate & {$[\mathrm{M}-\mathrm{H}]^{-}$} & 0.2 \\
\hline 269.2486 & Heptadecanoic acid & {$[\mathrm{M}-\mathrm{H}]^{-}$} & 0.0 \\
\hline 279.2325 & Linoleic acid & {$[\mathrm{M}-\mathrm{H}]^{-}$} & -0.4 \\
\hline 281.2481 & Oleic acid & {$[\mathrm{M}-\mathrm{H}]^{-}$} & -0.5 \\
\hline 283.2637 & Stearic acid & {$[\mathrm{M}-\mathrm{H}]^{-}$} & -0.6 \\
\hline 295.2270 & Hydroxyoctadecadienoic acid & {$[\mathrm{M}-\mathrm{H}]^{-}$} & -0.8 \\
\hline 303.2324 & Arachidonic acid & {$[\mathrm{M}-\mathrm{H}]^{-}$} & -0.6 \\
\hline 305.2479 & Eicosatrienoic acid & {$[\mathrm{M}-\mathrm{H}]^{-}$} & -0.7 \\
\hline 327.2325 & Eicosatrienoic acid & {$[\mathrm{M}+\mathrm{Na}-2 \mathrm{H}]^{-}$} & 1.9 \\
\hline 329.2480 & Docosapentaenoic acid & {$[\mathrm{M}-\mathrm{H}]^{-}$} & -0.6 \\
\hline 367.1590 & Dehydroepiandrosterone sulfate & {$[\mathrm{M}-\mathrm{H}]^{-}$} & 0.5 \\
\hline 369.1746 & Androsterone sulfate & {$[\mathrm{M}-\mathrm{H}]^{-}$} & 0.4 \\
\hline 377.2468 & Tetracosahexaenoic acid & {$[\mathrm{M}+\mathrm{Na}-2 \mathrm{H}]^{-}$} & 0.6 \\
\hline 383.1537 & Dihydroxyandrostenone sulfate & {$[\mathrm{M}-\mathrm{H}]^{-}$} & 0.3 \\
\hline 391.2258 & $\operatorname{LPA}(16: 0)$ & {$\left[\mathrm{M}-\mathrm{H}-\mathrm{H}_{2} \mathrm{O}\right]^{-}$} & 0.8 \\
\hline 409.2367 & LPA (16:0) & {$[\mathrm{M}-\mathrm{H}]^{-}$} & 0.7 \\
\hline 417.2419 & LPA $(18: 1)$ & {$\left[\mathrm{M}-\mathrm{H}-\mathrm{H}_{2} \mathrm{O}\right]^{-}$} & 1.3 \\
\hline 419.2572 & LPA (18:0) & {$\left[\mathrm{M}-\mathrm{H}-\mathrm{H}_{2} \mathrm{O}\right]^{-}$} & 0.9 \\
\hline 435.2524 & LPA (18:1) & {$[\mathrm{M}-\mathrm{H}]^{-}$} & 0.7 \\
\hline 437.2683 & LPA (18:0) & {$[\mathrm{M}--\mathrm{H}]^{-}$} & 0.9 \\
\hline 459.2532 & LPA (18:0) & {$[\mathrm{M}+\mathrm{Na}-2 \mathrm{H}]^{-}$} & 3.9 \\
\hline 465.3056 & Cholesterol sulfate & {$[\mathrm{M}-\mathrm{H}]^{-}$} & 1.2 \\
\hline 480.3106 & LPE (18:0) & {$[\mathrm{M}-\mathrm{H}]^{-}$} & 1.0 \\
\hline 508.3418 & LPE (20:0) & {$[\mathrm{M}-\mathrm{H}]^{-}$} & 1.0 \\
\hline 581.3113 & LPI (18:0) & {$\left[\mathrm{M}-\mathrm{H}-\mathrm{H}_{2} \mathrm{O}\right]^{-}$} & 2.2 \\
\hline 598.4625 & $\operatorname{CerP}(34: 1)$ & {$\left[\mathrm{M}-\mathrm{H}-\mathrm{H}_{2} \mathrm{O}\right]^{-}$} & 2.4 \\
\hline 599.3215 & LPI (18:0) & {$[\mathrm{M}-\mathrm{H}]^{-}$} & 1.3 \\
\hline 616.4731 & CerP (34:1) & {$[\mathrm{M}-\mathrm{H}]^{-}$} & 2.0 \\
\hline 644.5063 & $\operatorname{CerP}(36: 1)$ & {$[\mathrm{M}-\mathrm{H}]^{-}$} & 3.8 \\
\hline 671.4672 & PA (34:2) & {$[\mathrm{M}-\mathrm{H}]^{-}$} & 1.4 \\
\hline 673.4837 & PA $(34: 1)$ & {$[\mathrm{M}-\mathrm{H}]^{-}$} & 2.3 \\
\hline
\end{tabular}




$\begin{array}{lc}695.4671 & \text { PA }(36: 4) \\ 699.4993 & \text { PA }(36: 2) \\ 721.4828 & \text { PA }(36: 2) \\ 726.5818 & \text { CerP }(42: 1) \\ 742.5399 & \text { PE }(36: 2) \\ 744.5558 & \text { PE }(36: 1) \\ 766.5404 & \text { PE }(38: 4) \\ 768.5567 & \text { PE }(38: 3) \\ 770.5719 & \text { PE }(38: 2) \\ 778.5158 & \text { ST }(34: 1) \\ 794.5105 & \text { PE }(42: 9) \\ 795.5151 & \text { PG }(36: 2) \\ 885.5504 & \text { PI }(38: 4)\end{array}$

$\begin{array}{cc}{[\mathrm{M}-\mathrm{H}]^{-}} & 1.3 \\ {[\mathrm{M}-\mathrm{H}]^{-}} & 2.3 \\ {[\mathrm{M}+\mathrm{Na}-2 \mathrm{H}]^{-}} & 3.8 \\ {[\mathrm{M}-\mathrm{H}]^{-}} & 1.1 \\ {[\mathrm{M}-\mathrm{H}]^{-}} & 0.7 \\ {[\mathrm{M}-\mathrm{H}]^{-}} & 0.9 \\ {[\mathrm{M}-\mathrm{H}]^{-}} & 1.2 \\ {[\mathrm{M}-\mathrm{H}]^{-}} & 1.8 \\ {[\mathrm{M}-\mathrm{H}]^{-}} & 1.4 \\ {[\mathrm{M}-\mathrm{H}]^{-}} & 1.4 \\ {\left[\mathrm{M}-\mathrm{H}-\mathrm{H}{ }_{2} \mathrm{O}\right]^{-}} & -1.9 \\ {[\mathrm{M}+\mathrm{Na}-2 \mathrm{H}]^{-}} & -0.6 \\ {[\mathrm{M}-\mathrm{H}]^{-}} & 0.5\end{array}$




\section{REFERENCES}

(1) Psychogios, N.; Hau, D. D.; Peng, J.; Guo, A. C.; Mandal, R.; Bouatra, S.; Sinelnikov, I.; Krishnamurthy, R.; Eisner, R.; Gautam, B.; Young, N.; Xia, J. G.; Knox, C.; Dong, E.; Huang, P.; Hollander, Z.; Pedersen, T. L.; Smith, S. R.; Bamforth, F.; Greiner, R.; McManus, B.; Newman, J. W.; Goodfriend, T.; Wishart, D. S. Plos One 2011, 6, e16957. 